Pacific Journal of Mathematics

A CHARACTERIZATION OF FREE PROJECTIVE PLANES

Vol. 15, No. 1

September 1965 


\section{A CHARACTERIZATION OF FREE PROJECTIVE PLANES}

\section{C. Siebenmann}

A characterization of free projective planes is given that is more symmetrical than the original definition of M. Hall. It tends to very simple proofs of two fundamental theorems due to $M$. Hall and $L$. I. Kopejkina-one being the result that every subplane of a free plane is free.

In a fundamental paper Marshall Hall defines a free plane to be a projective plane which either is degenerate or is generated as follows from a 'basis configuration,' $\pi_{0}$, consisting of at least two points on a line together with two isolated points. For each pair of points not already joined in $\pi_{0}$ create a distinct line that joins them and add it to $\pi_{0}$. In the resulting configuration, $\pi_{1}$, consider pairs of lines that do not intersect, and for each create a distinct intersection point and add it to $\pi_{1}$, thus forming $\pi_{2}$. Continuing, construct $\pi_{3}, \pi_{4}, \pi_{5}$, $\pi_{6}$, etc. adding alternately lines and points as indicated above. Then $\pi=\bigcup_{n} \pi_{n}$ (with the obvious incidence relation) is a projective plane. It is by definition a free plane. Hall proved that a free plane contains no confined configuration, that is, no finite configuration that, like the Desargues configuration, has $\geqq 3$ points on each line and $\geqq 3$ lines through each point. Further, using a complicated argument, he showed that, if a finitely generated plane contains no confined configuration, it is free. It follows that any finitely generated subplane of a free plane is free.

L. I. Kopejkina [2] proved, shortly after, that an arbitrary subplane of a free plane is free. (Of interest is the analogy with free groups.) An exposition of Kopejkina's theorem appears in [3].

Because it suggests a more symmetrical definition of free plane that leads to very direct proofs of the above theorems, we introduce the notion of an extension process.

DeFinition 1. An extension process is a well ordered nested sequence of partial planes $\pi_{0} \subset \pi_{1} \subset \cdots \subset \pi_{n} \subset \cdots$ (the subscripts $0,1, \cdots, n, \cdots$ belonging to a well ordered set) such that if a point $p$ and a line $l$ appear in a term $\pi_{n}, n>0$, and in no earlier term then $p$ is not incident with $l$-in other words, the new elements in $\pi_{n}$ may be incident in $\pi_{n}$ with elements appearing in earlier terms, but have no incidences among themselves. From this point we adopt as definition the characterization of free plane we aim to justify. 
Definition 2. A free plane is a (possibly degenerate) projective plane, $\pi$, for which there exists an extension process $\pi_{0} \subset \pi_{1} \subset \ldots$ $\subset \pi_{n} \subset \cdots$ with $\pi_{0}=\varnothing$ (the empty plane) and $\pi=\bigcup_{n} \pi_{n}$ such that every new point [or line] in a term $\pi_{n}$ is incident in $\pi_{n}$ with at most two lines [points].

Immediately we can prove

Theorem I (Kopejkina). Every subplane of a free plane is free.

Proof. If $\pi$ is a free plane and $\pi_{0} \subset \pi_{1} \subset \cdots \subset \pi_{n} \subset \cdots$ is an extension process as above for $\pi$, then, given any subplane $\pi^{\prime}$, the sequence $\pi_{0} \cap \pi^{\prime} \subset \pi_{1} \cap \pi^{\prime} \subset \cdots \subset \pi_{n} \cap \pi^{\prime} \subset \cdots$ is visibly such an extension process for $\pi^{\prime}$. So $\pi^{\prime}$ is free.

Some definitions and notations are collected in $\S 2$. In $\S 3$ the result of Hall is proved. Our definition of a free plane is apparently broader than M. Hall's. In $\S 4$ we prove that the definitions are equivalent.

2. A set of elements consisting of points and lines, together with a relation of incidence between points and lines is said to form a partial plane (or configuration) if every two distinct lines [points] are together incident with at most one point [respectively line], (which when it exists we call their join). If every two distinct lines [points] are together incident with exactly one point [respectively line] the system becomes a (projective) plane. A plane is said to be nondegenerate if it contains 4 points no 3 of which are incident with the same line, and otherwise is said to be degenerate.

If $\rho$ and $\sigma$ are subpartial planes of the partial plane $\pi$, then $\rho+\sigma$ (or $\rho \cup \sigma$ ), $\rho \cap \sigma$, and $\rho-\sigma$ are subpartial planes of $\pi$ defined in the obvious way.

A configuration $\rho$ in a plane $\pi$ is said to generate the least subplane containing $\rho$. This subplane is denoted by $[\rho]_{\pi}$ (or $[\rho]$ ) and is called the completion of $\rho$ in $\pi$. The plane $\pi$ is finitely generated if, for some finite configuration $\rho \subset \pi,[\rho]=\pi$.

An extension process, $\mathscr{E}$, is regularly presented in the form $\mathscr{E}=$ $\left\{\pi_{n} ; n \in N\right\}$ where $N=\{0,1, \cdots, n, \cdots\}$ is a well ordered set. $\pi_{n-}$ will denote $\bigcup_{m<n} \pi_{m}$. $\mathscr{E}$ is said to act on $\pi_{0}$ and have the result $\mathscr{E}\left(\pi_{0}\right)=$ $\mathrm{U}_{n} \pi_{n}$. It should be pointed out that the partial plane $\mathscr{E}\left(\pi_{0}\right)$ need not be a full projective plane. The $\mathscr{E}$-stage (or simply stage) of an element $x \in \mathscr{E}\left(\pi_{0}\right)$ is the least $n$ such that $x \in \pi_{n}$. If $n>0, x$ is said to appear at $\mathscr{E}$-stage $n$; it is incident in $\pi_{n}$ with certain elements called its $\mathscr{E}$-bearers (or bearers), and these must all lie in $\pi_{n-}=\mathbf{U}_{m<n} \pi_{m}$. Elements in $\pi_{0}$ (by convention) have no bearers. Observe that if $x \notin \pi_{0}$ 
is incident with $y$, and $y$ is not a bearer of $x$, then $x$ must be a bearer of $y$, for they cannot both appear at the same stage.

If $\rho$ is a subpartial plane of $\pi=\mathscr{E}\left(\pi_{0}\right)$, there is a naturally defined restriction of $\mathscr{E}$ to $\rho$, denoted $\mathscr{E} \cap \rho$, viz. $\left\{\pi_{n} \cap \rho ; n \in N\right\}$. This is apparently a process that acts on $\pi_{0} \cap \rho$ with result $\rho$. Also there is a naturally defined saturation of $\mathscr{E}$ by $\rho$, denoted $\mathscr{E}+\rho$, viz. $\left\{\pi_{n}+\rho ; n \in N\right\}$. It apparently acts on $\pi_{0}+\rho$ with result $\pi$. We make two simple but important observations.

(1) The bearers in $\mathscr{E} \cap \rho$ of an element $x \in \rho$ are just those $\mathscr{E}$ bearers that lie in $\rho$.

(2) The bearers in $\mathscr{E}+\rho$ of an element $x \notin \pi_{0}+\rho$ include its $\mathscr{E}$-bearers and in addition any elements of $\rho$ incident with $x$ in $\pi$.

If $\mathscr{E}$ and $\mathscr{F}$ are extension processes, $\mathscr{E}$ acting on $\pi_{0}$ and $\mathscr{F}$ acting on $\mathscr{E}\left(\pi_{0}\right)$, then there is a naturally defined composition of $\mathscr{F}$ with $\mathscr{E}$ denoted $\mathscr{F} \circ \mathscr{E}$.

We call a process, $\mathscr{E}$, (a) bound; (b) free; (c) hyperfree if for all $n>0$ every new element of $\pi_{n}$ has (a) $\geqq 2$; (b) 2 ; (c) $\leqq 2$ bearers. Of course an extension process need not fall into any of these categories. A free plane is by definition a plane which is the result of a hyperfree process acting on the empty plane, $\varnothing$.

A bound process $\mathscr{E}$, whose result, $\mathscr{E}\left(\pi_{0}\right)$, is a full projective plane, is called a completion process for $\pi_{0}$. If $\rho$ is a configuration in a plane $\pi$, there is a canonical completion process $\mathscr{E}=\left\{\rho_{n} ; n \in J^{+}\right\}$, indexed on the integers $\geqq 0$, that acts on $\rho$ with result $[\rho]_{\pi}$. In fact $\rho_{0}=\rho$ and $\rho_{n}$ is defined inductively as the subpartial plane of $\pi$ whose elements are those of $\rho_{n-1}$ together with all points [lines] of $\pi$ that are joins of lines [or points] of $\rho_{n-1}$ resp. as $n$ is even or odd.

3. Now we prove Hall's result. Suppose $\pi$ is a finite partial plane having $P$ points, $L$ lines, and $I$ incidences between the points and lines. The rank of $\pi$ as defined by Hall is

$$
r(\pi)=2(P+L)-I \text {. }
$$

LemMa 1. Any finite partial plane, $\pi$, which contains no confined configurations is the result of a hyperfree process.

Proof. Set $\pi_{m}=\pi$ where $m$ is the number of points and lines in $\pi$. Since $\pi_{m}$ is not confined, there exists some element $x_{m} \in \pi_{m}$ which is incident with $\leqq 2$ elements in $\pi_{m}$. Define $\pi_{m-1} \subset \pi_{m}$ to be $\pi_{m}$ less the element $x_{m}$. Since $\pi_{m-1}$ is not confined, the process may be repeated, and after exactly $m$ steps we obtain $\pi_{0}=\varnothing$. Then $\mathscr{F}=$ $\left\{\pi_{i} ; i=0,1, \cdots, m\right\}$ is hyperfree and $\mathscr{F}(\varnothing)=\pi$. 
CoRollary. The rank of a finite partial plane containing no confined configuration is nonnegative.

\section{Proof. In fact $r(\pi) \geqq r\left(\pi_{m}\right) \geqq \cdots \geqq r\left(\pi_{0}\right)=0$.}

THeOREM II (Hall). A free plane contains no confined configuration. A finitely generated plane that contains no confined configuration is free.

REMARK. Kopejkina [2] constructed a plane (not finitely generated) that contains no confined configuration, but is not free.

Proof. Suppose first that $\pi$ is a free plane and $\mathscr{F}=\left\{\pi_{n} ; n \in N\right\}$ is a hyperfree process such that $\mathscr{F}(\varnothing)=\pi$. If $\rho$ is any finite configuration in $\pi$, there is an element $x \in \rho$ having maximal $\mathscr{F}$-stage, $m$. Since $x$ is incident with at most two elements of $\pi_{m}$, it obviously cannot be incident with at least three elements of $\rho \subset \pi_{m}$. Thus $\rho$ cannot be a confined configuration.

The proof of the second assertion depends essentially on our definition of free plane. Suppose that the plane $\pi$ is generated by a finite configuration $\pi_{0}$ and that $\pi$ contains no confined configuration. Let $\mathscr{E}=\left\{\pi_{n} ; n \in J^{+}\right\}$be the canonical completion process for $\pi_{0}$. Observe that each partial plane $\pi_{n}$ is finite and $\mathscr{E}\left(\pi_{0}\right)=\pi$. Since $\mathscr{E}$ is bound $r\left(\pi_{0}\right) \geqq r\left(\pi_{1}\right) \geqq \cdots \geqq r\left(\pi_{n}\right)$. But by the corollary above $r\left(\pi_{n}\right) \geqq 0$ for all $n$. Hence for some integer $m$ the minimal rank be attained, and thereafter $r\left(\pi_{n}\right)$ will have this minimal value. But this means that in the bound process $\mathscr{S}=\left\{\pi_{n} ; n \geqq m\right\}$ every element has exactly 2 bearers i.e., $\mathscr{S}$ is free. Now, by Lemma $1, \pi_{m}=\mathscr{T}\left(\pi_{0}\right)$ where $\mathscr{T}$ is hyperfree. So composing $\mathscr{S}$ with $\mathscr{T}$ we obtain a hyperfree process with $\mathscr{S} \circ \mathscr{T}(\varnothing)=\pi$.

4. This last section is devoted to proving that the adopted definition of free plane is equivalent to Hall's.

Lemma 2. Suppose $\mathscr{F}$ is hyperfree and $\pi=\mathscr{F}\left(\pi_{0}\right)$ is a plane. Then

(1) $\mathscr{F}_{1}=\mathscr{F} \cap\left[\pi_{0}\right]$ is a free completion process for $\pi_{0}$ in $\pi$; in fact, for $x \in\left[\pi_{0}\right]$, the $\mathscr{F}$-bearers lie in $\left[\pi_{0}\right]$ and coincide with those in any completion process for $\pi_{0}$ in $\pi$.

(2) $\mathscr{F}_{2}=\mathscr{F}+\left[\pi_{0}\right]$ is still hyperfree; in fact, for $x \notin\left[\pi_{0}\right]$, the $\mathscr{F}_{2}$-bearers coincide with the $\mathscr{F}$-bearers.

Proof. (1) Let $\mathscr{E}$ be any completion process for $\pi_{0}$ in $\pi$. If the first assertion is false, let $x$ be an element of least $\mathscr{E}$-stage, $m$, for 
which the $\mathscr{E}$-bearers do not conincide with the $\mathscr{F}$-bearers. Clearly $m>0$. Then at least one of the $\geqq 2 \mathscr{E}$-bearers of $x$, say $y$, must fail to be an $\mathscr{F}$-bearer. Since $x \notin \pi_{0}, y$ must have $x$ as an $\mathscr{F}$-bearer. But $y$ doesn't have $x$ as an $\mathscr{E}$-bearer and $y$ has $\mathscr{E}$-stage $<m$. This is a contradiction.

(2) Supposing the second assertion false, we have some $x \notin\left[\pi_{0}\right]$ with an $\mathscr{F}_{2}$-bearer $y$ that is not an $\mathscr{F}$-bearer and (hence) lies in $\left[\pi_{0}\right]-\pi_{0}$. But $x$ is incident with $y \notin \pi_{0}$; so $x \notin\left[\pi_{0}\right]$ must be an $\mathscr{F}$ bearer of $y \in\left[\pi_{0}\right]$ in contradiction to (1).

REMARK. This lemma has a useful generalization in which $\pi_{0}$ is replaced in (1) and (2) by a partial plane $\rho \subset \pi_{0}$ that is 'complete' in $\pi_{0}$ (see $[1,4.3])$. Then, in the proof of (1), the possibility that $x \in \pi_{0}$ must be eliminated.

Definition 3. A free completion of a partial plane, $\pi_{0}$, is a plane $\pi$ which is the result of a free process acting on $\pi_{0}$.

Again this seems less restrictive than Hall's definition, but

Theorem III. Any two free completions of a partial plane $\pi_{0}$ are related by a unique isomorphism that fixes $\pi_{0}$.

Proof. Let $\mathscr{F}$ be a free completion process for $\pi_{0}$, and let $\mathscr{E}$ be the canonical completion process for $\pi_{0}$ in $\mathscr{F}\left(\pi_{0}\right)$. Clearly $\mathscr{E}\left(\pi_{0}\right)=$ $\left[\pi_{0}\right]=\mathscr{F}\left(\pi_{0}\right)$; and according to assertion (1) of the above lemma, $\mathscr{E}$ is free. The theorem now follows from the fact that, if $\mathscr{E}$ and $\mathscr{E}^{\prime}$ are two canonical free completion processes for $\pi_{0}$, there is a unique isomorphism of $\mathscr{E}\left(\pi_{0}\right)$ onto $\mathscr{E}^{\prime}\left(\pi_{0}\right)$ that fixes $\pi_{0}$.

In a free extension process $\left\{\pi_{0}, \pi_{1}\right\}$ of just two terms we say that $\pi_{1}$ is derived from $\pi_{0}$ by a free addition, and $\pi_{0}$ from $\pi_{1}$ by a free subtraction. Two partial planes are free equivalent if the one can be derived from the other by a finite sequence of free additions and subtractions. Free equivalent partial planes evidently have isomorphic free completions.

Recall that a basis configuration consists of a number of points on a 'base' line and two isolated points.

THEOREM IV. Every nondegenerate free plane contains a basis configuration of which it is a free completion.

Proof. Suppose $\pi=\mathscr{F}(\varnothing)$ is a free plane, where $\mathscr{F}=\left\{\pi_{n} ; n \in N\right\}$ is a hyperfree process. We may assume without loss of generality that at each stage one element and no more is added, i.e., $\pi_{n}=\pi_{n-}+x_{n}$, where $x_{n}$ is an element that has 0,1 , or 2 bearers. 
Applying Lemma 2 to $\mathscr{F}$ for stages $\geqq n$ we find that $\mathscr{F}^{\prime}=$ $\mathscr{F}+\left[\pi_{n-}\right]$ is hyperfree; applying the same lemma to $\mathscr{F}^{\prime}$ for stages $>n$ we find that $\mathscr{F}^{\prime} \cap\left[\pi_{n}\right]$ free completes $\left[\pi_{n-}\right]+x_{n}$ to $\left[\pi_{n}\right]$.

Now let $a$ be the first stage such that $\left[\pi_{a}\right]$ is nondegenerate. Then $\left[\pi_{a-}\right]$ must be a degenerate plane, and, as we have observed, $\left[\pi_{a}\right]$ is a free completion of $\left[\pi_{a-}\right]+x_{a}$. By an inspection of the various cases one shows that $\left[\pi_{a-}\right]+x_{a}$ is in every case free equivalent to some basis configuration $\pi_{0}^{\alpha}$. This implies that $\left[\pi_{a}\right]$ is a free completion of $\pi_{0}^{\alpha}$.

For $n>a$, one readily shows (cf. [1] or [3]) that $\left[\pi_{n-}\right]+x_{n}$ is free equivalent to $\left[\pi_{n-}\right]$ with a set $\mu_{n}$ of points adjoined to the base line, $l$, of $\pi_{0}^{\alpha}$. (The set $\mu_{n}$ consists of 2 or 1 points if $x_{n}$ is incident with 0 or 1 elements in $\left[\pi_{n-}\right]$, and of course $\mu_{n}$ is empty when $x_{n} \in\left[\pi_{n-}\right]$.) Thus $\left[\pi_{n}\right]$ is a free completion of $\left[\pi_{n-}\right]+\mu_{n}$.

We will show that $\pi$ is a free completion of the basis configuration $\pi_{0}^{\beta}=\pi_{0}^{\alpha}+\bigcup_{n>a} \mu_{n}$. Let $\left\{\left(\left[\pi_{n-}\right]+\mu_{n}\right)^{k} ; k \in J^{+}\right\}$be the canonical process that free completes $\left[\pi_{n-}\right]+\mu_{n}$ to $\pi_{n}$, and form the composition of all these processes to obtain $\mathscr{S}=\left\{\left(\left[\pi_{n-}\right]+\mu_{n}\right)^{k} ;(n, k) \in N \times J^{+}, n \geqq a\right\}$ where $\left(\left[\pi_{a-}\right]+\mu_{a}\right)$ is to be read as $\pi_{0}^{\alpha}$, and $N \times J^{+}$is well ordered lexicographically. This is a hyperfree process acting on $\pi_{0}^{\alpha}$ with result $\pi$. The saturation $\overline{\mathscr{S}}=\mathscr{S}+\pi_{0}^{\beta}$ is the desired free completion process.

Clearly $\overline{\mathscr{S}}\left(\pi_{0}^{\beta}\right)=\pi$; and $\overline{\mathscr{S}}$ is a bound process since all elements with $<2 \mathscr{S}$-bearers lie in $\pi_{0}^{\beta}$. To show that $\overline{\mathscr{S}}$ is actually free observe that every new element, $x$, of $\overline{\mathscr{S}}$ appeared in $\mathscr{S}$ with exactly 2 bearers. If $x$ has an extra bearer, $y$, in $\overline{\mathscr{S}}$, then $y \in \mu_{n}$ where $x$ has $\overline{\mathscr{S}}$-stage $(m, k) \in N \times J^{+}$and $n>m$. But $y$ is incident with both $x$ and base line $l$ of $\pi_{0}^{\alpha}$, i.e., $y$ is the join of $x$ and $l$. Then $y \in\left[\pi_{m}\right]$ in contradiction to $y \in \mu_{n}$. So $\overline{\mathscr{S}}$ must be free.

This completes the proof that the definition of free plane we have proposed coincides with M. Hall's definition.

\section{REFERENCES}

1. M. Hall, Projective planes, Trans. Amer. Math. Soc. 54 (1943), 229-277.

2. L. I. Kopejkina, Decomposition of projective planes, Bull. Acad. Sci. USSR, Ser. Math. Izvestia Akad. Nauk, SSSR), 9 (1945), 495-526.

3. L. A. Skornyakov, Projective planes, Trans. Amer. Math. Soc. Series 1, 1 (1962), 51-107. 


\section{PACIFIC JOURNAL OF MATHEMATICS}

\section{EDITORS}

H. Samelson

Stanford University

Stanford, California

R. M. Blumenthal

University of Washington

Seattle, Washington 98105
J. Dugundu

University of Southern California Los Angeles, California 90007

*Richard Arens

University of California

Los Angeles, California 90024

\section{ASSOCIATE EDITORS}
E. F. BECKENBACH
B. H. NeumanN
F. WOLF
K. YOSIDA

\section{SUPPORTING INSTITUTIONS}

UNIVERSITY OF BRITISH COLUMBIA CALIFORNIA INSTITUTE OF TECHNOLOGY UNIVERSITY OF CALIFORNIA MONTANA STATE UNIVERSITY

UNIVERSITY OF NEVADA

NEW MEXICO STATE UNIVERSITY

OREGON STATE UNIVERSITY

UNIVERSITY OF OREGON

OSAKA UNIVERSITY

UNIVERSITY OF SOUTHERN CALIFORNIA
STANFORD UNIVERSITY

UNIVERSITY OF TOKYO

UNIVERSITY OF UTAH

WASHINGTON STATE UNIVERSITY

UNIVERSITY OF WASHINGTON

AMERICAN MATHEMATICAL SOCIETY CALIFORNIA RESEARCH CORPORATION SPACE TECHNOLOGY LABORATORIES NAVAL ORDNANCE TEST STATION 


\section{Pacific Journal of Mathematics}

\section{Vol. 15, No. $1 \quad$ September, 1965}

Donald Charles Benson, Unimodular solutions of infinite systems of linear

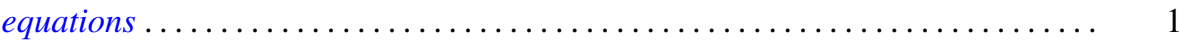

Richard Earl Block, Transitive groups of collineations on certain designs . . . . . . 13

Barry William Boehm, Existence of best rational Tchebycheff approximations .... . 19

Joseph Patrick Brannen, A note on Hausdorff's summation methods . . . . . . . . . . 29

Dennison Robert Brown, Topological semilattices on the two-cell ............ 35

Peter Southcott Bullen, Some inequalities for symmetric means . . . . . . . . . . 47

David Geoffrey Cantor, On arithmetic properties of coefficients of rational

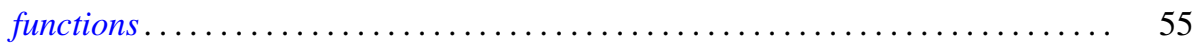

Luther Elic Claborn, Dedekind domains and rings of quotients . . . . . . . . . 59

Allan Clark, Homotopy commutativity and the Moore spectral sequence ........ 65

Allen Devinatz, The asymptotic nature of the solutions of certain linear systems of

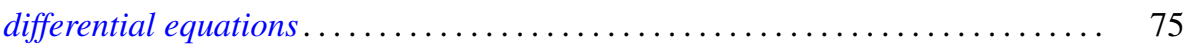

Robert E. Edwards, Approximation by convolutions ................... 85

Theodore William Gamelin, Decomposition theorems for Fredholm operators . . . . . 97

Edmond E. Granirer, On the invariant mean on topological semigroups and on

topological groups .................................. 107

Noel Justin Hicks, Closed vector fields . . . . . . . . . . . . . . . . . . . 141

Charles Ray Hobby and Ronald Pyke, Doubly stochastic operators obtained from

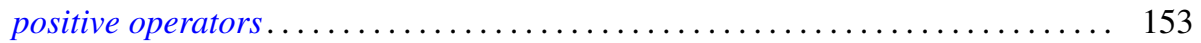

Robert Franklin Jolly, Concerning periodic subadditive functions . . . . . . . . . 159

Tosio Kato, Wave operators and unitary equivalence . . . . . . . . . . . . . . 171

Paul Katz and Ernst Gabor Straus, Infinite sums in algebraic structures . . . . . . . 181

Herbert Frederick Kreimer, Jr., On an extension of the Picard-Vessiot theory ...... 191

Radha Govinda Laha and Eugene Lukacs, On a linear form whose distribution is

identical with that of a monomial ......................... 207

Donald A. Ludwig, Singularities of superpositions of distributions . . . . . . . . . 215

Albert W. Marshall and Ingram Olkin, Norms and inequalities for condition

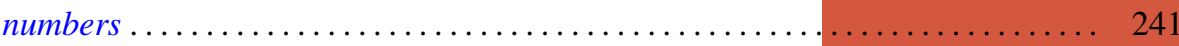

Horace Yomishi Mochizuki, Finitistic global dimension for rings . . . . . . . . . . 249

Robert Harvey Oehmke and Reuben Sandler, The collineation groups of division

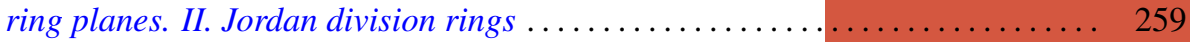

George H. Orland, On non-convex polyhedral surfaces in $E^{3} \ldots \ldots \ldots \ldots \ldots \ldots \ldots 267$

Theodore G. Ostrom, Collineation groups of semi-translation planes . . . . . . . . 273

Arthur Argyle Sagle, On anti-commutative algebras and general Lie triple

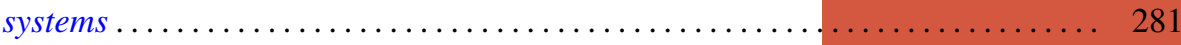

Laurent Siebenmann, A characterization of free projective planes . . . . . . . . . 293

Edward Silverman, Simple areas.................................. 299

James McLean Sloss, Chebyshev approximation to zero .................. 305

Robert S. Strichartz, Isometric isomorphisms of measure algebras . . . . . . . . . 315

Richard Joseph Turyn, Character sums and difference sets . . . . . . . . . . . . 319

L. E. Ward, Concerning Koch's theorem on the existence of arcs . . . . . . . . . . 347

Israel Zuckerman, A new measure of a partial differential field extension ......... 357 